\title{
Observation of energy transfer at optical frequency to an ultrathin silicon waveguide
}

\author{
LIPING FANG, ${ }^{1,}{ }^{*}$ LEFTERIS DANOS, ${ }^{2}$ TOM MARKVART, ${ }^{3,4}$ RUI CHEN ${ }^{1, *}$ \\ ${ }^{1}$ Department of Electrical and Electronic Engineering, Southern University of Science and Technology, Shenzhen 518055, China \\ ${ }^{2}$ Department of Chemistry, Energy Lancaster, Lancaster University, Lancaster LA1 4YB, UK \\ ${ }^{3}$ Centre for Advanced Photovoltaics, Czech Technical University, Prague 16627, Czech Republic \\ ${ }^{4}$ Solar Energy Laboratory, Faculty of Engineering and Physical Sciences, University of Southampton, Southampton SO17 1BJ, UK \\ *Corresponding author: liping.fang@outlook.com, chenr@sustech.edu.cn
}

Received XX Month XXXX; revised XX Month, XXXX; accepted XX Month XXXX; posted XX Month XXXX (Doc. ID XXXXX); published XX Month XXXX

\begin{abstract}
Energy transfer from a submonolayer of rhodamine 6G molecules to a $130 \mathrm{~nm}$ thick crystalline silicon waveguide is investigated. The dependence of fluorescence lifetime of rhodamine on its distance to the silicon waveguide is characterized and modeled successfully by a classical dipole model. The energy transfer process could be regarded as photon tunneling into the silicon waveguide via the evanescent waves. The experimentally observed tunneling rate is well described by an analytical expression obtained via complex variable analysis in the complex wavenumber plane. (c) 2020 Optical Society of America
\end{abstract}

http://dx.doi.org/10.1364/OL.99.099999

Energy transfer between organic light absorber and proximal inorganic semiconductor such as silicon (Si) is attracting the attention of researchers $[1,2]$. The combined merits of organic and inorganic materials offer the possibility to fabricate optoelectronic devices with unprecedented properties [3]. The incident radiation on the organic absorber could be funneled into the inorganic material via energy transfer. Energy transfer at optical frequencies to a nearby waveguide has been investigated theoretically $[4,5]$ and experimentally [6]. The observed energy transfer via a Förster-type resonance energy transfer for distances close to the waveguide surface (up to $10 \mathrm{~nm}$ ) has been studied using quantum dots [6]. In this paper we focus on coupling to the evanescent waves for medium distances $(10-50 \mathrm{~nm})$ with the use of molecular dipoles, allowing a finer distance resolution. We have termed this evanescent coupling process as 'photon tunneling' [7] and we present a detailed distance dependent study and theoretical model to fit our results. We analyze, in the complex wavenumber plane, the time-resolved fluorescence resulting from energy transfer from a submonolayer of rhodamine $6 \mathrm{G}$ molecules to an ultrathin $\mathrm{Si}$ waveguide. This leads us to propose a novel photon management technique which allows the detection of photon tunneling into individual waveguide modes.
As shown in Fig. 1, all the samples (size of $15 \mathrm{~mm} \times 15 \mathrm{~mm}$ ) were fabricated on a silicon-on-insulator (SOITECH) substrate. The thickness of the Si device layer and the silicon dioxide $\left(\mathrm{SiO}_{2}\right)$ box layer was $130 \pm 5 \mathrm{~nm}$ and $1934 \pm 5 \mathrm{~nm}$, respectively. Variable thickness spacer layer between rhodamine and the Si device layer was $\mathrm{SiO}_{2}$ film deposited by plasma-enhanced chemical vapor deposition (PECVD, Oxford Instruments PlasmaPro80). The rhodamine submonolayer was obtained by spin-coating of a few drops of dilute alcohol solution of rhodamine $6 \mathrm{G}\left(3 \times 10^{-4} \mathrm{~mol} / \mathrm{L}\right)$ on the substrate with the speed of 3000 round per minute for 60 seconds [8]. To improve the stability of rhodamine, a final capping layer of polymethyl methacrylate (PMMA) with thickness around $70 \mathrm{~nm}$ was deposited on all the samples by spin-coating of a few drops of $2 \%$ anisole solution of PMMA (MicroCHEM NANO 950PMMA). The thickness and refractive index of the $\mathrm{SiO}_{2}$ spacer layer and the PMMA capping layer were characterized by spectroscopic ellipsometry using a Horiba UVISEL phasemodulated ellipsometer. PMMA was chosen due to its similar refractive index with the $\mathrm{SiO}_{2}$ spacer layer in the concerned spectral range, thereby providing a homogeneous optical environment for the rhodamine molecules.

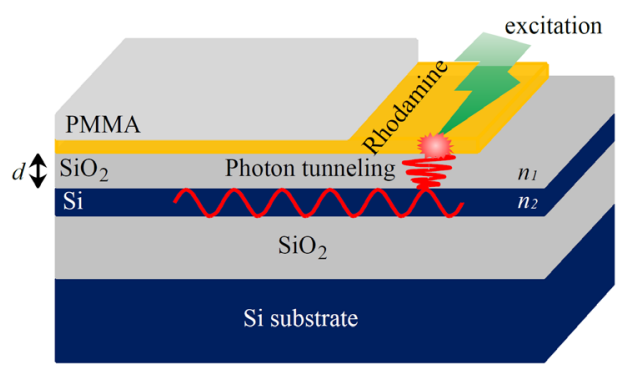

Fig. 1. Sample structure of energy transfer from submonolayer rhodamine to an ultrathin silicon waveguide.

Fluorescence lifetime was measured by a time-resolved fluorescence spectrometer (PicoQuant FluoTime 300) which 
employed the time-correlated single photon counting (TCSPC) technique. The excitation source was a $512 \mathrm{~nm}$ diode laser (PicoQuant LDH-P-C-520) working at $40 \mathrm{MHz}$ repetition rate. Fluorescence emission was collected by a high numerical aperture collecting lens, passed through a $532 \mathrm{~nm}$ long-pass edge filter (Semrock LP03-532RE-25) and captured by a single photon detector module (PicoQuant PMA-C 192-N-M) equipped with a grating monochromator (Bentham MSH300). This monochromator was set at $600 \mathrm{~nm}$, i.e. fluorescence emission was monitored at this wavelength to avoid reabsorption or intermolecular energy transfer, which would complicate the analysis of energy transfer to Si. Fluorescence decay curves were collected by TCSPC via the TimeHarp 260 module. The obtained decays were fitted with the sum of two exponential functions after deconvolution of the instrument response function (IRF), which was obtained by measuring the scattering decay curve of the pulsed laser at the excitation wavelength. The longer lifetime was attributed to rhodamine, whereas the shorter one $(1.1 \pm 0.5 \mathrm{~ns}$, similar for all rhodamine-Si distance) was attributed to PMMA [9]. Chance, Prock and Silbey (CPS) developed a classical model for molecular fluorescence near interface [10]. The excited fluorescent molecules were modeled as forced and damped electric dipoles. The normalized damping rate (inverse fluorescence lifetime) of an electric dipole emitting near a single interface is given by:

$$
\begin{gathered}
\hat{b}_{\mathrm{VED}}=q \operatorname{Re}\left(\frac{3}{2 k_{1}^{3}} \int_{0}^{\infty} d k_{\rho} \frac{k_{\rho}^{3}}{k_{1 x}}\left\{r_{12}^{\mathrm{TM}} \exp \left(i 2 k_{1 x} d\right)+\frac{1}{q}\right\}\right) \\
\hat{b}_{\mathrm{HED}}=q \operatorname{Re}\left\{\begin{array}{l}
\frac{3}{4 k_{1}^{3}} \int_{0}^{\infty} d k_{\rho} k_{1 x} k_{\rho}\left\{-r_{12}^{\mathrm{TM}} \exp \left(i 2 k_{1 x} d\right)+\frac{1}{q}\right\}+ \\
\frac{3}{4 k_{1}} \int_{0}^{\infty} d k_{\rho} \frac{k_{\rho}}{k_{1 x}}\left\{r_{12}^{\mathrm{TE}} \exp \left(i 2 k_{1 x} d\right)+\frac{1}{q}\right\}
\end{array}\right\}
\end{gathered}
$$

where $\hat{b}_{\mathrm{VED}}$ and $\hat{b}_{\mathrm{HED}}$ represent the damping rate of a vertical electric dipole (VED) and a horizontal electric dipole (HED), respectively, normalized to the damping rate without the presence of the interface. $q$ is the fluorescence quantum yield of the molecule, $k_{\rho}$ and $k_{i x}$ are the in and out of plane wavenumbers in region $i$ and defined by $k_{i}^{2}=k_{\rho}^{2}+k_{i x}^{2}, d$ is the distance between the dipole and the interface, $r_{12}^{\mathrm{TE}}$ and $r_{12}^{\mathrm{TM}}$ are the Fresnel reflection coefficients for the transverse electric (TE) and transverse magnetic (TM) waves at the interface between region 1 and region 2, respectively. For dipole emission near a thin waveguide, these two reflection coefficients are given by Airy's summation:

$$
r=\frac{-r_{21}+r_{23} e^{i 2 \phi}}{1-r_{21} r_{23} e^{i 2 \phi}}
$$

where $r_{i j}$ represents the Fresnel reflection coefficient for the TE/TM waves at the interface between region $i$ and region $j$, $\phi=k_{2 x} t$ the phase retardation for light travelling through the thin waveguide with thickness $t$. For an isotropic distribution of dipole orientations, the damping rate is given by $\hat{b}_{\mathrm{ISO}}=\left(\hat{b}_{\mathrm{VED}}+2 \hat{b}_{\mathrm{HED}}\right) / 3$.

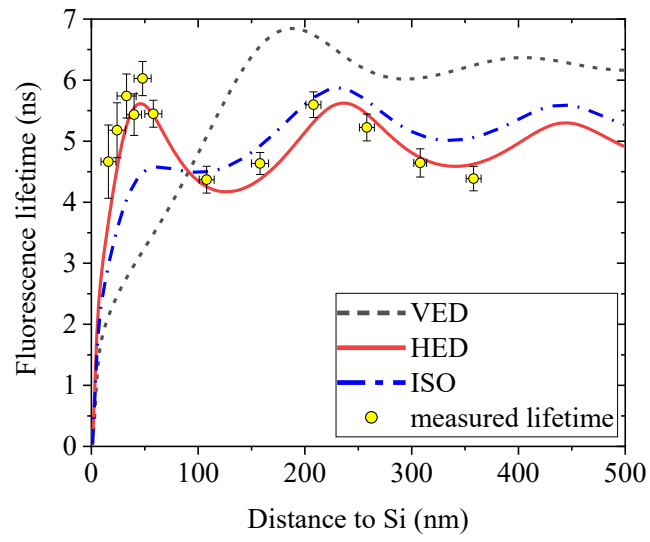

Fig. 2. Comparison of fluorescence lifetime with CPS model for different dipole orientation configurations.

The dependence of fluorescence lifetime of rhodamine on its distance to the Si waveguide surface is illustrated in Fig. 2. The measured fluorescence lifetime can be well fitted by the CPS model for a HED, i.e. Eq. (2), using the following refractive index for Si and $\mathrm{SiO}_{2}: n_{1}=n_{3}=n_{\mathrm{SiO}_{2}}=1.458, n_{2}=n_{\mathrm{Si}}=3.940+0.020 \mathrm{i}$ [11]. The horizontal dipole orientation for rhodamine $6 \mathrm{G}$ molecules rest on flat substrates has been revealed by previous angular fluorescence intensity [12] and fluorescence lifetime investigations [13].

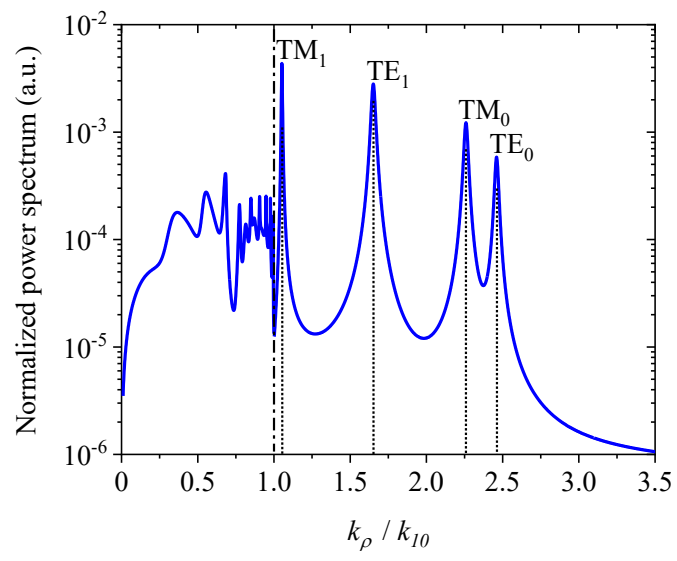

Fig. 3. Power spectrum of a HED emitting near the silicon waveguide.

Ford and Weber [14] introduced a power spectrum $d \mathscr{P} / d k_{\rho}$ to define the total power $\mathscr{P}$ dissipated by the dipole emitter via $\mathscr{P}=\int_{0}^{\infty} d k_{\rho}\left(d \mathscr{P} / d k_{\rho}\right)$. Since $\mathscr{P}$ is the product of photon energy and the dipole damping rate, $d \mathscr{P} / d k_{\rho}$ is directly proportional to the integrand of the dipole damping rate given by Eq. (1) or Eq. (2). The radiated power in the far field can be obtained by integrating $d \mathscr{P} / d k_{\rho}$ in the range $0<k_{\rho}<k_{10}$. The power transferred to Si can be calculated in the range $k_{10}<k_{\rho}<k_{20}$. Note that this part of energy is evanescent in region 1 , but propagating in region 2 . We have termed this evanescent coupling process as 'photon tunneling', through the potential barrier which confines photons inside the high refractive index material by total internal 
reflection [7]. The power spectrum of a HED emitting near the structure shown in Fig. 1 is plotted in Fig. 3. There are four distinct peaks in the range $k_{\rho}>k_{10}$. We find that these peaks correspond to the waveguide modes supported by the Si waveguide, after we solved the dispersion relation (as shown in Fig. 4) of the structure shown in Fig. 1 numerically.

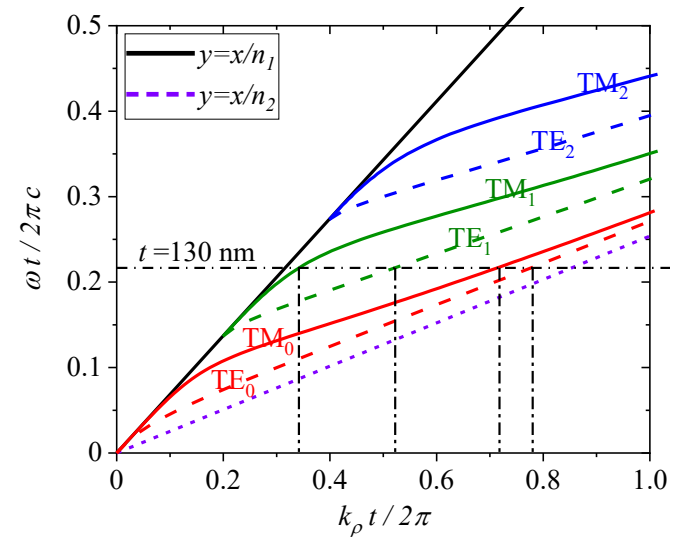

Fig. 4. Dispersion relation of the silicon waveguide.

Equation (3) has singularities when the denominator approaches zero. These singularities (slightly off the real axis) are the poles in the complex plane of the in-plane wavenumber, which physically corresponds to the waveguide modes supported by the $\mathrm{Si}$ waveguide. Analytical expressions were obtained to approximate the tunneling rate to these modes. We first expand the denominator of Eq. (3) in the vicinity of the pole in the complex in-plane wavenumber plane, then perform contour integral along a semicircle with infinitesimal radius around that pole. The photon tunneling rate for VED and HED are found to be:

$$
\begin{aligned}
\hat{b}_{\mathrm{VED}}^{p t} \approx & \sum_{m=0}^{M} \frac{3 \pi}{2 k_{1}^{3}} \frac{k_{\rho m}^{3}}{\left|k_{1 x m}\right|} \frac{g_{\mathrm{TM}}\left(k_{\rho m}\right)}{f_{\mathrm{TM}}\left(k_{\rho m}\right)} \exp \left(-2\left|k_{1 x m}\right| d\right) \\
\hat{b}_{\mathrm{HED}}^{p t} \approx & \sum_{m=0}^{M} \frac{3 \pi}{4 k_{1}^{3}}\left|k_{1 x m}\right| k_{\rho m} \frac{g_{\mathrm{TM}}\left(k_{\rho m}\right)}{f_{\mathrm{TM}}\left(k_{\rho m}\right)} \exp \left(-2\left|k_{1 x m}\right| d\right) \\
& +\sum_{m=0}^{N} \frac{3 \pi}{4 k_{1}} \frac{k_{\rho m}}{\left|k_{1 x m}\right|} \frac{g_{\mathrm{TE}}\left(k_{\rho m}\right)}{f_{\mathrm{TE}}\left(k_{\rho m}\right)} \exp \left(-2\left|k_{1 x m}\right| d\right)
\end{aligned}
$$

where $M$ and $N$ are the number of TM and TE modes supported by the waveguide, $g_{\mathrm{TM}}$ and $f_{\mathrm{TM}}$ are given by

$$
\begin{aligned}
& g_{\text {TM }}\left(k_{\rho}\right)=\frac{4 \varepsilon_{1} \varepsilon_{2}\left|k_{1 x}\right| k_{2 x}}{\varepsilon_{2}^{2}\left|k_{1 x}\right|^{2}+\varepsilon_{1}^{2} k_{2 x}^{2}} \\
& f_{\mathrm{TM}}\left(k_{\rho}\right)=2 k_{\rho}\left\{\begin{array}{l}
\frac{t}{k_{2 x}}+\frac{\varepsilon_{1} \varepsilon_{2}\left(\left|k_{1 x}\right|^{2}+k_{2 x}^{2}\right)}{\varepsilon_{1}^{2}\left|k_{1 x}\right| k_{2 x}^{3}+\varepsilon_{2}^{2}\left|k_{1 x}\right|^{3} k_{2 x}} \\
+\frac{\varepsilon_{3} \varepsilon_{2}\left(\left|k_{3 x}\right|^{2}+k_{2 x}^{2}\right)}{\varepsilon_{3}^{2}\left|k_{3 x}\right| k_{2 x}^{3}+\varepsilon_{2}^{2}\left|k_{3 x}\right|^{3} k_{2 x}}
\end{array}\right\}
\end{aligned}
$$

where $\varepsilon_{i}$ is the dielectric constant of region $i$ which, when omitted in Eqs. (6) and (7), yields $g_{\mathrm{TE}}$ and $f_{\mathrm{TE}}$. The tunneling rates given by Eqs. (4) and (5) are the summation of exponential functions which correspond to the contribution of the individual waveguide modes. Equation (5) is calculated numerically and compared with the observed tunneling rate (obtained by subtracting the far field emission rate into the total damping rate) in Fig. 5. The solid curves signify the contribution of each waveguide mode. It is depicted that the modeled tunneling rate fits well with the observed data.

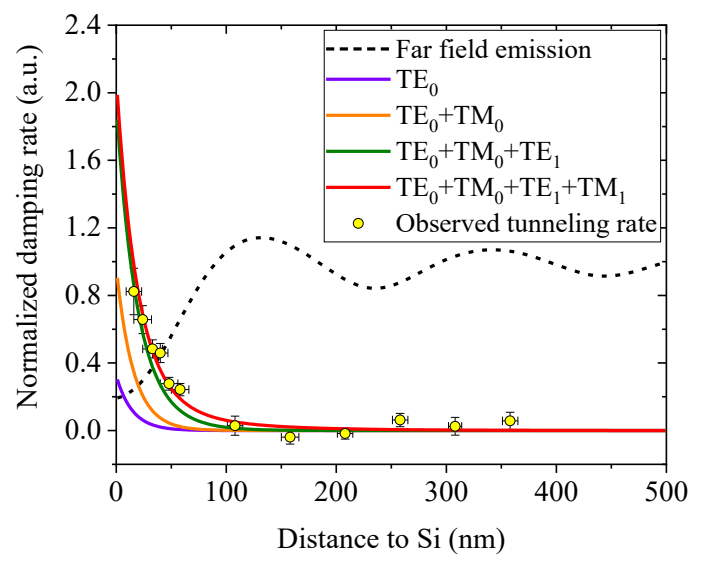

Fig. 5. Comparison of modeled and observed photon tunneling rates.

Energy transfer from a molecular emitter to an ultrathin Si waveguide is demonstrated in this work. The excited molecules transfer their excitonic energy to the underlying Si waveguide via photon tunneling. This is different from the Förster-type resonance energy transfer, which is only observed for emitter-Si distances less than about $5 \mathrm{~nm}[1,2]$. Through a complex variable analysis, analytical expressions have been found to successfully approximate the observed tunneling rates. The emission transition dipole of the excited rhodamine molecule is parallel to the surface of the $\mathrm{Si}$ substrate, consistent with previous angular fluorescence intensity measurements.

The energy transfer efficiency is an important factor in comparing different power dissipation channels of the excited molecules. Based on the ratio between the photon tunneling rate and the rate of fluorescence emission, the energy transfer efficiency is estimated to be $80 \%$ in our experiment. Ultimately, the coupling efficiency has to include reverse tunneling, based on which the molecules couple the power out of the waveguide via the evanescent waves. We would like to revisit this topic in a subsequent publication. The above experiments and theoretical analysis are important to the design and optimization of hybrid optoelectronic devices based on a photon management technique that allows the evanescent coupling of photons into individual waveguide modes.

Funding. National Natural Science Foundation of China (61604138); Czech Ministry of Education, Youth and Sport (CZ.02.1.01/0.0/0.0/15_003/0000464). 
Acknowledgment. PECVD and ellipsometry were performed using the tools maintained by SUSTECH Core Research Facilities. We thank technical help from Dr. X. Gao, Dr. R. Li, Mr. Z. Wang.

Disclosures. The authors declare no conflicts of interest.

\section{References}

1. N. Alderman, L. Danos, L. Fang, M. C. Grossel, and T. Markvart, Chem. Commun. 53, 12120 (2017).

2. L. Danos, N. R. Halcovitch, B. Wood, H. Banks, M. P. Coogan, N. Alderman, L. Fang, B. Dzurnak, and T. Markvart, Faraday Discuss., (2020).

3. W. Peng, S. M. Rupich, N. Shafiq, Y. N. Gartstein, A. V. Malko, and Y. J. Chabal, Chem. Rev. 115, 12764 (2015).

4. B. J. Soller, H. R. Stuart, and D. G. Hall, Opt. Lett. 26, 1421 (2001).

5. N. R. Verhart, G. Lepert, A. L. Billing, J. Hwang, and E. A. Hinds, Opt. Express 22, 19633 (2014).

6. H. M. Nguyen, O. Seitz, W. Peng, Y. N. Gartstein, Y. J. Chabal, and A. V. Malko, ACS Nano 6, 5574 (2012).

7. L. Fang, K. S. Kiang, N. P. Alderman, L. Danos, and T. Markvart, Opt. Express 23, A1528 (2015).

8. T. F. Heinz, C. K. Chen, D. Ricard, and Y. R. Shen, Phys. Rev. Lett. 48, 478 (1982).

9. L. Gaudreau, K. J. Tielrooij, G. E. D. K. Prawiroatmodjo, J. Osmond, F. J. G. de Abajo, and F. H. L. Koppens, Nano Lett. 13, 2030 (2013).

10. R. R. Chance, A. Prock, and R. Silbey, Adv. Chem. Phys. 37, 1 (1978).

11. M. A. Green, Sol. Energy Mater. Sol. Cells 92, 1305 (2008).

12. H. Sano, G. Mizutani, and S. Ushioda, Surf. Sci. 223, 621 (1989).

13. G. Cnossen, K. E. Drabe, and D. A. Wiersma, J. Chem. Phys. 98, 5276 (1993).

14. G. W. Ford, and W. H. Weber, Phys. Rep. 113, 195 (1984).

\section{Full Reference}

[1] N. Alderman, L. Danos, L. Fang, M. C. Grossel, and T. Markvart, "Light harvesting in silicon(111) surfaces using covalently attached protoporphyrin IX dyes," Chemical Communications, vol. 53, no. 89, pp. 12120-12123, 2017. https://doi.org/10.1039/C7CC04767C

[2] L. Danos, N. R. Halcovitch, B. Wood, H. Banks, M. P. Coogan, N. Alderman, L. Fang, B. Dzurnak, and T. Markvart, "Silicon photosensitisation using molecular layers," Faraday Discussions, 2020.

https://doi.org/10.1039/C9FD00095J

[3] W. Peng, S. M. Rupich, N. Shafiq, Y. N. Gartstein, A. V. Malko, and Y. J. Chabal, "Silicon surface modification and characterization for emergent photovoltaic applications based on energy transfer," Chemical Reviews, vol. 115, no. 23, pp. 12764-12796, 2015. https://doi.org/10.1021/acs.chemrev.5b00085

[4] B. J. Soller, H. R. Stuart, and D. G. Hall, "Energy transfer at optical frequencies to silicon-on-insulator structures," Optics Letters, vol. 26, no. 18, pp. 1421-1423, 2001. https://doi.org/10.1364/OL.26.001421

[5] N. R. Verhart, G. Lepert, A. L. Billing, J. Hwang, and E. A. Hinds, "Single dipole evanescently coupled to a multimode waveguide," Optics Express, vol. 22, no. 16, pp. 19633-19640, 2014. https://doi.org/10.1364/OE.22.019633
[6] H. M. Nguyen, O. Seitz, W. Peng, Y. N. Gartstein, Y. J. Chabal, and A. V. Malko, "Efficient radiative and nonradiative energy transfer from proximal CdSe/ZnS nanocrystals into silicon nanomembranes," ACS Nano, vol. 6, no. 6, pp. 5574-5582, 2012. http://dx.doi.org/10.1021/nn301531b

[7] L. Fang, K. S. Kiang, N. P. Alderman, L. Danos, and T. Markvart, "Photon tunneling into a single-mode planar silicon waveguide," Optics Express, vol. 23, no. 24, pp. A1528-A1532, 2015. https://doi.org/10.1364/OE.23.0A1528

[8] T. F. Heinz, C. K. Chen, D. Ricard, and Y. R. Shen, "Spectroscopy of molecular monolayers by resonant second-harmonic generation," Physical Review Letters, vol. 48, no. 7, pp. 478-481, 1982. https://doi.org/10.1103/PhysRevLett.48.478

[9] L. Gaudreau, K. J. Tielrooij, G. E. D. K. Prawiroatmodjo, J. Osmond, F. J. G. de Abajo, and F. H. L. Koppens, "Universal distance-scaling of nonradiative energy transfer to graphene," Nano Letters, vol. 13, no. 5, pp. 2030-2035, 2013. https://doi.org/10.1021/nl400176b

[10] R. R. Chance, A. Prock, and R. Silbey, "Molecular fluorescence and energy transfer near interfaces," Advances in Chemical Physics, vol. 37, no. pp. 1-65, 1978. https://doi.org/10.1002/9780470142561.ch1

[11] M. A. Green, "Self-consistent optical parameters of intrinsic silicon at 300K including temperature coefficients," Solar Energy Materials and Solar Cells, vol. 92, no. 11, pp. 1305-1310, 2008. https://doi.org/10.1016/j.solmat.2008.06.009

[12] H. Sano, G. Mizutani, and S. Ushioda, "Dipole radiation pattern from surface adsorbed dye molecules: Effects of surface roughness," Surface Science, vol. 223, no. 3, pp. 621-631, 1989. https://doi.org/10.1016/0039-6028(89)90686-9

[13] G. Cnossen, K. E. Drabe, and D. A. Wiersma, "Fluorescence properties of submonolayers of rhodamine $6 \mathrm{G}$ in front of a mirror," Journal of Chemical Physics, vol. 98, no. 7, pp. 5276-5280, 1993. https://doi.org/10.1063/1.464927

[14] G. W. Ford and W. H. Weber, "Electromagnetic interactions of molecules with metal surfaces," Physics Reports, vol. 113, no. 4, pp. 195-287, 1984. https://doi.org/10.1016/0370-1573(84)90098-X 